\title{
Gut microbiota and fecal metabolites in captive and wild North China leopard (Panthera pardus japonensis) by comparsion using $16 \mathrm{~s}$ rRNA gene sequencing and LC/ MS-based metabolomics
}

\author{
Yan Hua ${ }^{1,2 \dagger}$, Heqin $\mathrm{CaO}^{1 \dagger}$, Jiao Wang ${ }^{2}$, Fengping $\mathrm{He}^{3}$ and Guangshun Jiang ${ }^{1 *}$
}

\begin{abstract}
Background: Gut microbes significantly contribute to nutrient digestion and absorption, intestinal health and immunity, and are essential for the survival and environmental adaptation of wild animals. However, there are few studies on the gut microbiota of captive and wild North China leopard (Panthera pardus japonensis).

Results: A total of 10 mainly bacterial phyla were identified in the fecal microbiota of North China leopard, Lachnoclostridium ( $p=0.003)$, Peptoclostridium $(p=0.005)$, Bacteroides $(p=0.008)$, Fusobacterium $(p=0.017)$ and Collinsella $(p=0.019)$ were significantly higher than those of wild North China leopard. Distinct differences in the fecal metabolic phenotypes of captive and wild North China leopard were found, such as content of I-methionine, n-acetyl-I-tyrosine, pentadecanoic acid and oleic acid. Differentially abundant gut microbes were associated with fecal metabolites, especially the bacteria in Firmicutes and Bacteroidetes, involved in the metabolism of N-acetyl-Lalanine and D-quinovose.
\end{abstract}

Conclusion: This study reports for the first time the differences in gut microbiota abundance between captive and wild North China leopard, as well as significant differences in fecal metabolic phenotypes between two groups.

Keywords: Fecal metabolites, gut microbiota, North China leopard, $16 \mathrm{~S}$ rRNA gene sequencing, metabolomics

\section{Background}

The North China leopard (Panthera pardus japonensis), also called the China leopard, is one of the three native leopard subspecies distributed in China. The North China leopard is only found in the eastern and central parts of China [1]. Due to habitat fragmentation, largescale forest reduction and poor habitat connectivity, the

\footnotetext{
* Correspondence: jgshun@126.com

${ }^{\dagger}$ Yan Hua and Heqin Cao contributed equally to this work.

${ }^{1}$ Feline Research Center of National Forestry and Grassland Administration, College of Wildlife and Natural Protected Area, Northeast Forestry University, 150040 Harbin, China

Full list of author information is available at the end of the article
}

distribution range and population size of the North China leopard have sharply decreased [2-4], and it has been listed as a national class I key protected animal. It has also been listed as endangered (EN) by the Red List of Biodiversity in China-Vertebrate Volume. China's Red Book of Endangered Animals classified it as Endangered (E); it was upgraded from Vulnerable (VU) in 2008 to Near Threatened (NT) by the IUCN, and has been listed in Appendix I by CITES.

Fortunately, with the strengthening of protection, the North China leopard population shows stable and an increasing trend. However, due to its limited habitat areas

(c) The Author(s). 2020 Open Access This article is licensed under a Creative Commons Attribution 4.0 International License, which permits use, sharing, adaptation, distribution and reproduction in any medium or format, as long as you give appropriate credit to the original author(s) and the source, provide a link to the Creative Commons licence, and indicate if changes were made. The images or other third party material in this article are included in the article's Creative Commons licence, unless indicated otherwise in a credit line to the material. If material is not included in the article's Creative Commons licence and your intended use is not permitted by statutory regulation or exceeds the permitted use, you will need to obtain permission directly from the copyright holder. To view a copy of this licence, visit http://creativecommons.org/licenses/by/4.0/. The Creative Commons Public Domain Dedication waiver (http://creativecommons.org/publicdomain/zero/1.0/) applies to the data made available in this article, unless otherwise stated in a credit line to the data. 
and diets, there is an obviously increased risk of attack by diseases, especially intestinal diseases, which threaten the health and life of animals [5]. Gut microbial diversity analyses based on leopard fecal samples should also be considered as important insights on conservation.

Many studies have shown that the gastrointestinal tracts of human beings and animals contains large and complex microbial communities [6], and changes in the gut microbiota have been shown to affect host metabolism and energy homeostasis [7]. 16S rRNA sequencing technology is widely used in the study of the composition and abundance of the human and animal gut microbiota $[8,9]$. Researchers have also revealed differences in the composition and structure of the gut microbiota between captive and wild animals, and the stability of the gut microbiota is closely related to the health of the population $[10,11]$. The food of carnivores usually contains a large amount of animal tissue, which provides a substrate for intestinal microorganisms to use for fermentation [12, 13]. Specific compositions of the gut microbiota are associated with variations in the host diet and physiological status [14, 15]. Therefore, the study on the gut microbiota diversity of carnivores with different diets and living environments can better reveal the differences in the functional contribution of the gut microbiota.

Furthermore, regarding the relationship between gut microbes and the host fecal metabolic phenotype, nontargeted metabolomics can be used to explore how gut microbes affect metabolic function. Metabolomics analysis can reveal the abnormal metabolism and metabolic pathways involved in complex diseases [16]. Nontargeted fecal metabolome studies have been used to unravel metabolic phenotypic variation associated with gut microflora disturbances during disease occurrence [17].

Facing the wild environments, the wild North China leopard coevolves with its surroundings to help it adapt to the wild environment. In contrast, captive individuals are faced with specific artificial feeding environments and dietary conditions that are different from the wild [18]. Under such conditions, the metabolism and behavior of captive North China leopard may change, thus affecting the composition, structure and function of their gut microbiota. The gut microbiota of captive and wild North China leopard, and the relationship between microbial community and fecal metabolism phenotype, is of great importance for the healthy development of the North China leopard population. In this study, we want to reveal differences in the gut microbiota of captive and wild leopard, and the relationships between the gut microbiota and the fecal metabolic phenotype using an analysis method combining 16S rRNA gene sequencing and LC-MS metabolomics, providing scientific guidance for the protection and breeding of leopard population.

\section{Results}

Gut microbiota of the North China leopard

Based on 16S rRNA gene sequencing, the fecal microbiota composition of captive and wild North China leopard was analyzed, and 39.01G of high-quality leopard base sequences without adaptor and DNA contamination was obtained.

A total of 10 bacterial phyla were identified in the fecal microbiota 16S rRNA sequencing (Fig. 1). Firmicutes (41\%), Bacteroidetes (17.8\%), Fusobacteria (14.3\%), Actinobacteria (13.8\%), Proteobacteria (11.5\%) and Saccharibacteria $(8.22 \%)$ were the main bacteria components of captive North China leopard. In the wild individuals, Firmicutes (34.3\%), Actinobacteria (23.4\%), Proteobacteria (21.8\%), Bacteroidetes (19.2\%) and Saccharibacteria $(8.98 \%)$ were the main components. The fecal microbial composition of captive and wild animals was significantly different for Fusobacteria $(p=0.036)$, and the relative abundance was significantly higher than that of the wild leopard, but there was no significant difference at other phylum levels.

At the genus level, 18 genera showed significant differences between captive and wild leopards (Fig. 2), and the relative abundance of 11 genera, including Lachnoclostridium $(p=0.003)$, Peptoclostridium $(p=0.005)$, Bacteroides $(p=0.008), \quad$ Fusobacterium $(p=0.017)$ and Collinsella $(p=0.019)$, were significantly higher than those of wild leopards. There were 21 dominant genera in captive leopards and 23 dominant genera in wild North China leopard. Fusobacterium, Bacteroides and Collinsella were the dominant genera in the captive leopards. The wild leopards were completely different, and Kurthia, Glutamicibacter, Pseudomonas and Lactobacillus were the dominant genera. There were 9 common dominant genera of both groups, including Bacteroides, Lactobacillus, Desulfovibrio, Escherichia Shigella, Lachnospiraceae _ NK4A136 _ group, bacterium_f_bacteroidales_s24-7_group, Uncultured _ bacterium _ $f_{-}$Lachnospiraceae, uncultured _ bacterium $f_{-}$ Erysipelotrichaceae and Clostridium_sensu_stricto_1.

\section{Fecal metabolic profiling of North China leopard}

The fecal metabolic profiles of North China leopard were acquired by LC-MS. In the OPLS-DA model, the metabolic curves were obviously different, and the results showed that there were obvious differences in fecal metabolites between captive and wild leopard (Fig. 3).

The metabolites were identified by MS/MS based on accurate molecular weights and fragment patterns, and by comparison to online databases such as the Human Metabolome Database (HMDB;http://www.hmdb.ca/), Biofluid Metabolites Database (http://metlin.scripps.edu) and Massbank (http://www.massbank.jp/). The results showed that there were a total of 31 metabolites with 


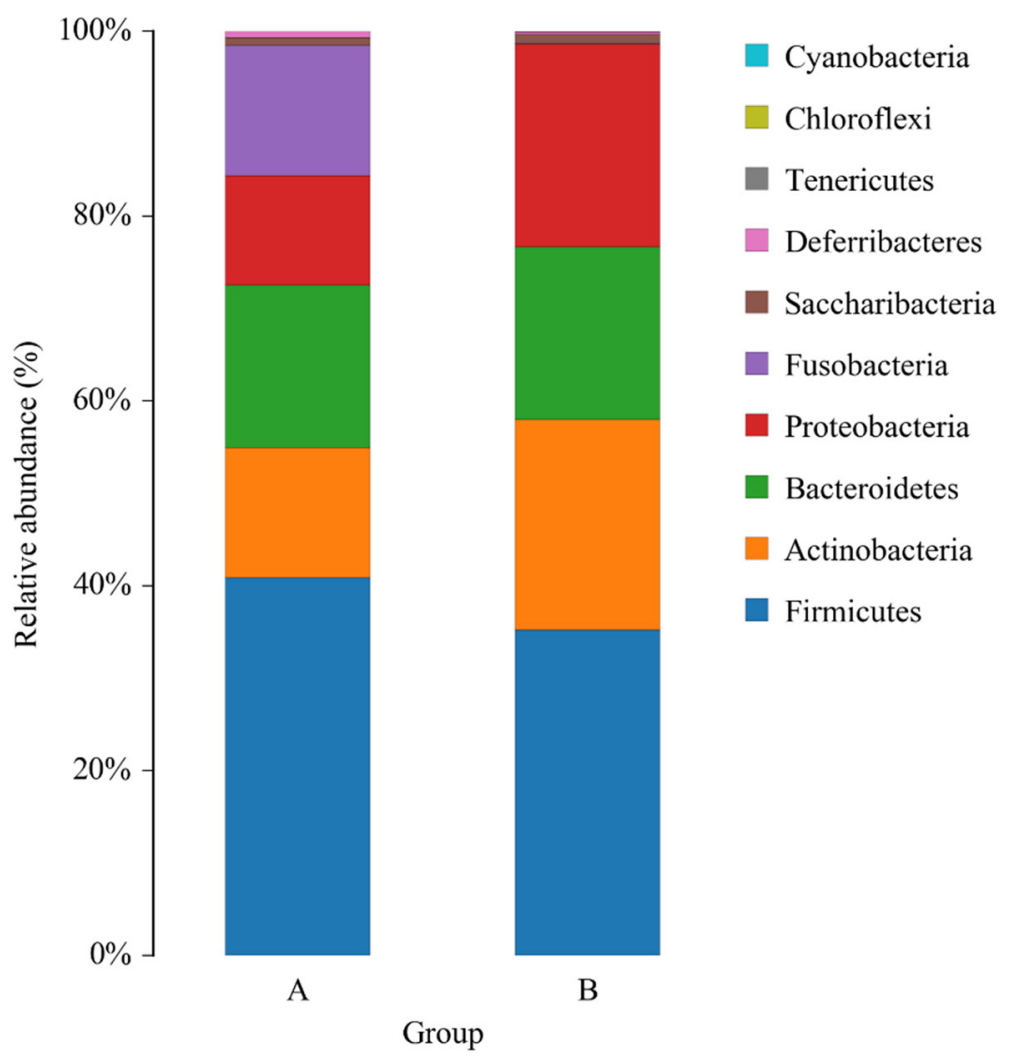

Fig. 1 Relative gut microbiota abundance at the phylum level in captive and wild North China leopard .Note: A group is the captive North China leopard, and B group is the wild North China leopard

significant differences between captive and wild North China leopard, and 9 metabolites with extremely significant differences (Fig. 4). The corresponding $P$ values and VIP values are shown in Table 1.

A heat map shows the trends of the metabolites of captive and wild leopard(Fig. 4), and a total of 18 metabolites were significantly higher in captive than wild leopard(N-acetyl-L-alanine, hydroxyhydroquinone, transcinnamate, L-methionine, isovalerylglycine, kynurenic acid, 3-indolepropionic acid, glycyl-L-leucine, $\mathrm{N}$-acetyldl-methionine, 1,3-dimethyluric acid, n-acetyl-l-tyrosine, d-quinovose, l-gulonic gamma-lactone, Dioxybenzone, 5-hydroxymethylcytidine, Salidroside, Ethynodiol diacetate, deoxycholic acid); 13 metabolites were significantly lower than that of wild leopard(vitamin L1, 5hydroxymethyluracil, Tiopronin, tetrahydrobiopterin, pentadecanoic acid, quadrone, oleic acid, stearic acid, beta-estradiol, eicosapentaenoic acid, l-iditol, tamsulosin, muramic acid).

\subsection{Correlation of gut microbiota with fecal metabolic phenotype of North China leopard}

To explore the correlation with significant differences in fecal metabolites and microorganisms between captive and wild leopards, we used the MetPA database, which is a part of MetaboAnalyst (www.metaboanalys.ca), and the Kyoto Encyclopedia of Genes and Genomes (KEGG) to analyze the pathway of the metabolites that differed between these two leopard groups (Fig. 5). The results showed that the relative abundances of KEGG firstorder metabolic pathways such as Metabolism, Genetic Information Processing and Human Diseases were the highest among the gut microbiota of both captive and wild leopards. The metabolites enriched in Biosynthesis of unsaturated fatty acids were significantly different, and the amount of different metabolites enriched in the metabolic pathways was the largest.

Spearman correlation analysis of significantly different metabolites and significantly different OTUS (microbes) was performed to obtain the relationships between metabolites and microbes (Fig. 6). In the overall network diagram, the bacteria in Firmicutes and Bacteroidetes were most closely related to metabolites, and the dominant microbes showed different positive or negative correlations to different metabolites.

In summary, the composition of the gut microbiota of captive and wild leopards was significantly different, as were the phenotypes of fecal metabolites. 


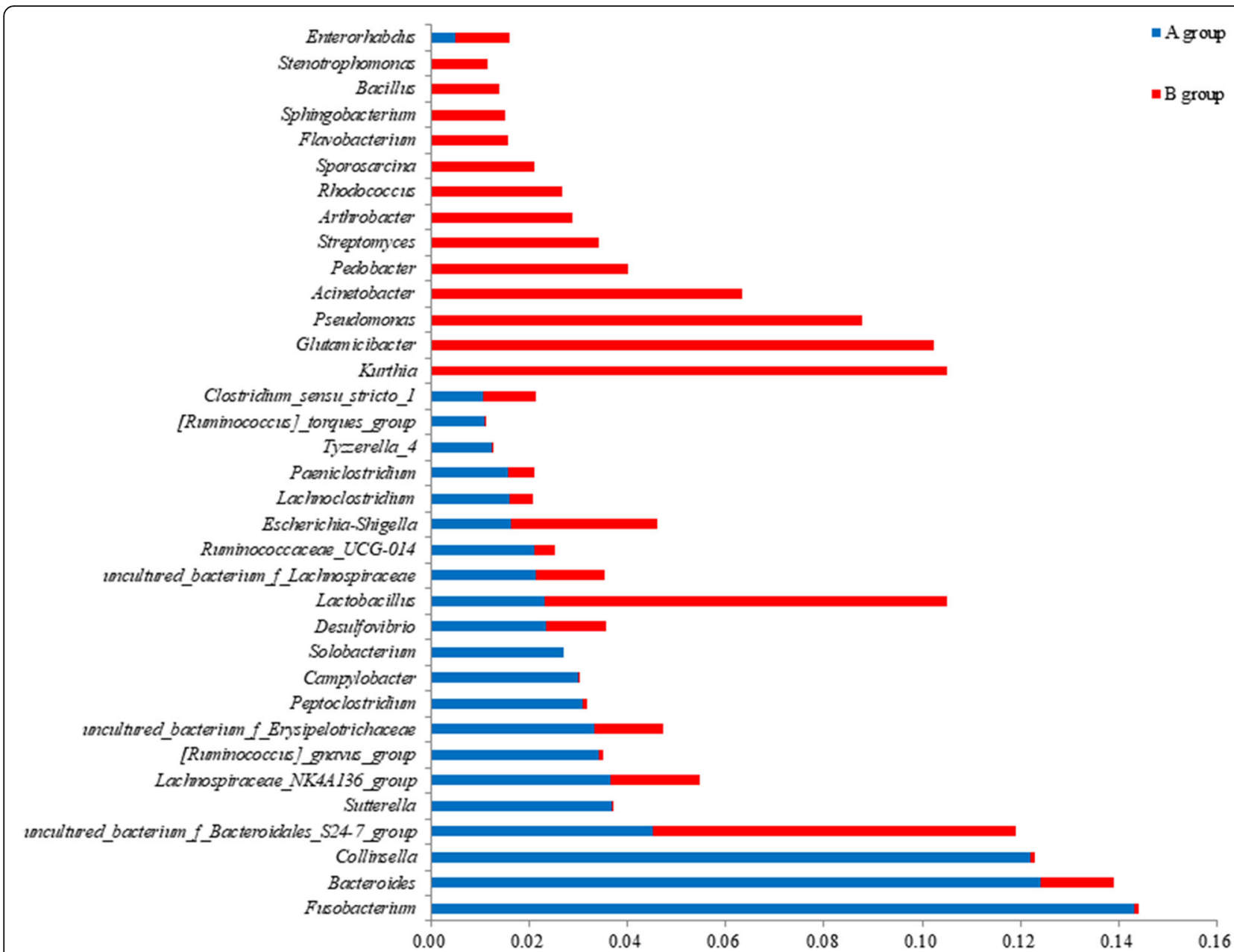

Fig. 2 Relative gut microbiota abundance at the genus level in captive and wild North China leopard. Note: A group is the captive North China leopard, and B group is the wild North China leopard

\section{Discussion}

\section{Gut microbiota of North China leopard and comparison} with those of other carnivores

This study suggested that five major phyla, Firmicutes, Bacteroides, Actinobacteria, Proteobacteria and Saccharibacteria were observed to be different in captive and wild leopard. In addition, Fusobacteria was the major phylum in captive leopard. Firmicutes, Bacteroides, Actinobacteria and Proteobacteria were the main microbes of the captive and wild leopards, consistent with the results of studies on other carnivores, such as snow leopard [19, 20], wolves[21], Amur tiger[22], Amur leopard and cheetahs [19-26]. Fecal samples of healthy domestic cats (Felis catus) [27] and snow leopard (Panthera uncia) [19, 20, 28] featured similar phylum compositions with slightly different proportions. In our study, the relative abundance of Fusobacteria of captive and wild leopards was significantly different $(p=0.036)$, but other phyla showed no significant differences. It has been reported that bacteria of the phylum Fusobacteria are not uniformly distributed in different mammals, the contents of Cheetahs (Acinonyx jubatus) [26], Amur leopard (Panthera pardus orientalis) [29], North China leopard [24], snow leopard [19], wolves(Canis lupus) [23] were different.

Firmicutes was the most dominant phylum in both captive and wild leopards and it showed no significant difference between the two groups $(p=0.526)$. Many studies have reported that Firmicutes is the most predominant phylum of animals [29-31] and humans [32]. Hildebrandt reported that a high-fat diet was associated with an increase in Firmicutes and a decrease in Bacteroidetes [33]. We observed that the proportion of Firmicutes in captive North China leopard was relatively more abundant than that in wild leopards, and the proportion of Bacteroidetes in captive leopards was relatively lower, which might be related to the fact that captive animals are fed a fixed amount of food every day, unlike wild animals which hunt by themselves, resulting in greater body fat storage for the captive population. 


\section{Scores (OPLS-DA)}

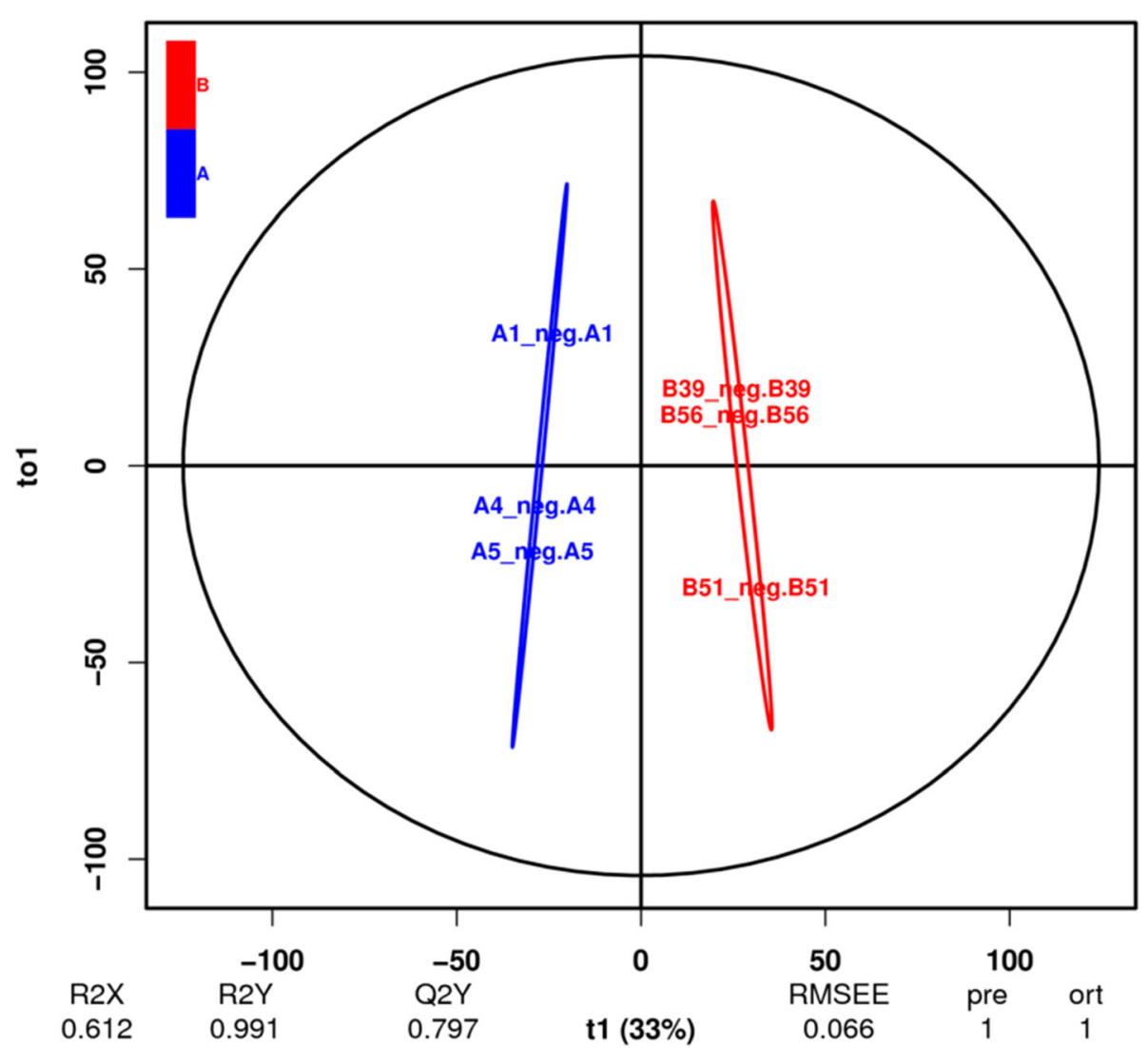

Fig. 3 OPLS-DA score of captive and wild North China leopard. Note: A1, A4 and A5 are captive North China leopard, B39, B51 and B56 are wild North China leopard

Actinobacteria account for a large proportion of the gut microbiota of captive (13. 8\%) and wild North China leopard (23.4\%), and there was no significant different between the two groups $(p=0.473)$. In snow leopard [19, 20], Amur tiger [29] and North China leopard (Panthera tigris altaica) [24], Actinobacteria was the predominant phylum. In contrast, Wu reported that Actinobacteria constituted $0.53 \%$ in wolves[34]. Our study may indicate that Actinobacteria is the dominant phylum in felines, but there might be differences between felines and canine species, and perhaps among different mammalian species.

The relative abundance of gut probiotics such as Lactobacillus and Bifidobacterium in the wild leopard is higher than that in captive leopard. Bifidobacterium and Lactobacillus can inhibit the growth of harmful bacteria, resist infection by pathogenic bacteria such as Staphylococcus, Shigella and Salmonella, decompose carcinogenic substances, and improve disease resistance[35]. These abilities are largely related to the adaptation of the wild North China leopard to complex life in the wild. Moreover, the relative abundance of pathogenic bacteria such as Shigella, Acinetobacter and Pseudomonas in the gut microbiota of wild North China leopard is higher than that of captive leopard. The gut microbiota is a dynamically balanced ecological network, and dynamic balance and stability among the host, food and intestinal bacteria are maintained through interactions. Once the balance and stability are broken, the health of the body will be seriously threatened [36]. Additionally, the species and content of pathogenic bacteria in the gut bacteria of wild North China leopard are higher than that of captive leopard. This might indicate that the ecological network of the gut microbiota of wild leopard is in a stable state and reveals that gut microbes coevolved with the host; gut microbes were influenced by the living environment and feeding habits of animals, also affecting the physiological functions of the host [18].

The relationships between fecal metabolites and diseases Concerning the metabolomics, existing disease data have revealed changes in metabolic pathways and metabolites in human and animal models [37-39]. In recent years, metabolomic studies of fecal samples have found that 


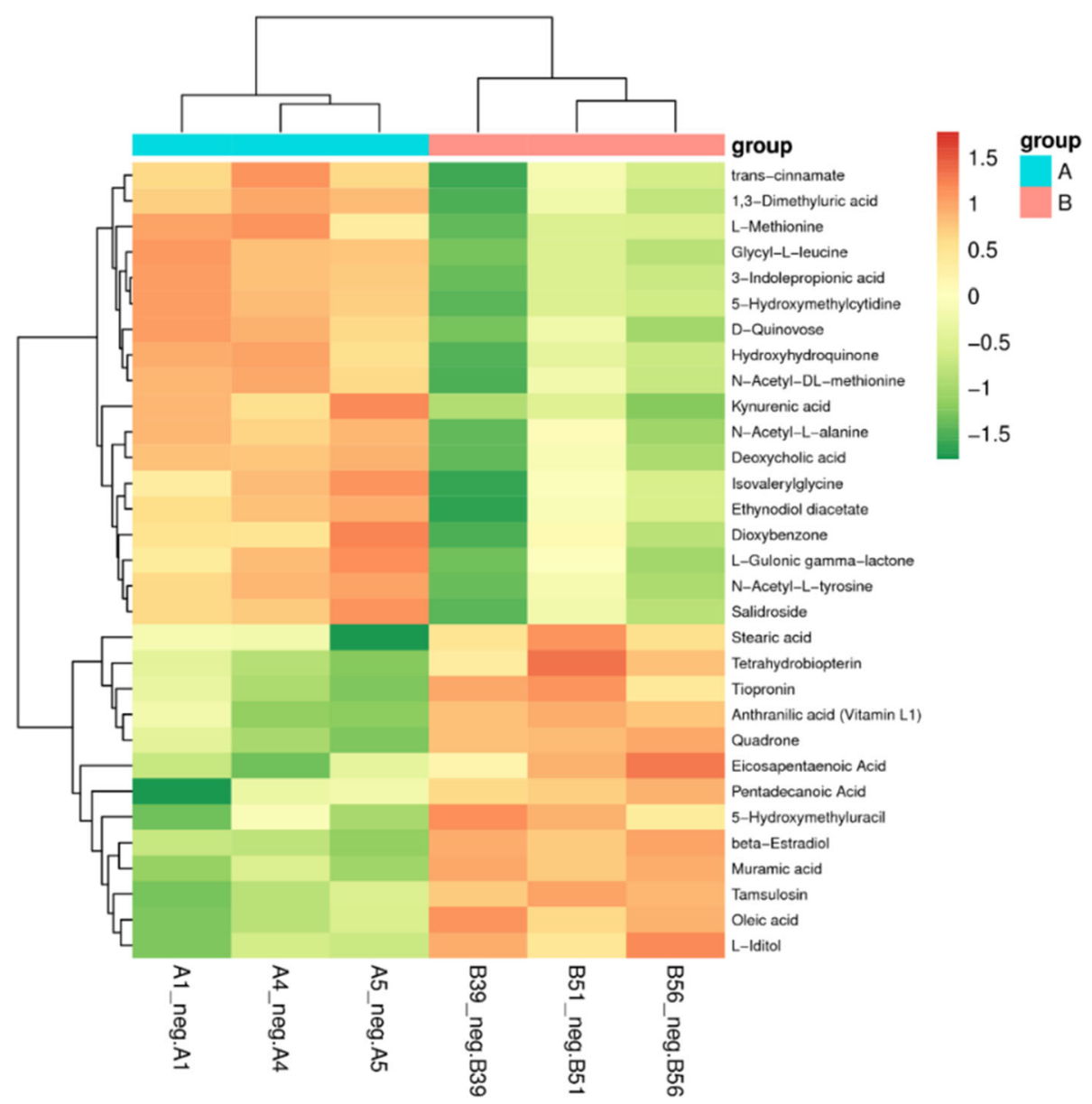

Fig. 4 Heat map of metabolites with significant differences based on LC/MS data of fecal samples. Red and green represent higher and lower concentrations of metabolites in captive and wild North China leopard, respectively

metabolic phenotypic changes are associated with gut microbiota changes in the development of diseases, such as obesity, Crohn's disease and colorectal cancer [40-42].Alkaline and neutral volatile metabolites such as indole and p-methylphenol are thought to be a toxin that causes blood clots, and increased bile acid is linked to an increased risk of colorectal cancer [43-45]. In our study, we found that the fecal metabolic profile of captive North China leopard was significantly different from that of wild leopards. The content of l-methionine in fecal metabolites of wild North China leopard was significantly lower than that of captive individuals. Methionine-supplementation was able to partially mitigate adverse effects caused by the higher stocking density and to improve redox status of the broilers [46], it may be a sign that the North China leopard has adapted to life in captivity. Lipid metabolism such as pentadecanoic acid, oleic acid altered in mustard airway diseases (MADs) patient [47], we found the variation of pentadecanoic acid and oleic acid on wild and captive North China leopard, but the reason for this result is not clear.

\section{Correlations between the gut microbiota and fecal} metabolites of captive and wild North China leopard

An increasing number of studies have shown that fecal metabolites are closely related to allergic diseases, endocrine, metabolic, and gastrointestinal diseases, which can not only reflect the gut microbe ecological state but also help to diagnose and predict the occurrence and development of diseases [48-50]. In our study, we observed significant correlations between the gut microbiota and fecal metabolites using Spearman correlation analysis. Interestingly, most bacteria belonging to Firmicutes are positively correlated with metabolites; for example, Firmicutes have a strong positive correlation with $\mathrm{N}$-acetylL-Alanine, trans-cinnamate, L-methionine, and hydroxyhydroquinone. In contrast, most bacteria belonging to Bacteroidetes are negatively correlated with metabolities; strong negative correlations were found with Dquinovose, deoxycholic acid, quadrone, and muramic acid.

Gut microbiota co-evolve with the host and adapt to different physiological and pathological states, and 
Table 1 Metabolites differences between captive and wild North China leopard achieved from the analyses of feces samples submitted to liquid chromatography/mass spectrometer (LC-MS) analyses using an Ultra High Performance Liquid Chromatography (UHPLC) system

\begin{tabular}{|c|c|c|c|c|}
\hline No. & Metabolites & $P$ value & VIP & Regulated \\
\hline 999 & $\mathrm{~N}$-acetyl-L-alanine & 0.006 & 1.602 & $U p^{b}$ \\
\hline 1155 & anthranilic acid (vitamin L1) & 0.004 & 1. 723 & Down $^{b}$ \\
\hline 1331 & hydroxyhydroquinone & 0.022 & 1. 595 & $U p^{a}$ \\
\hline 1474 & trans-cinnamate & 0.044 & 1. 534 & $U p^{a}$ \\
\hline 1492 & L-methionine & 0.049 & 1. 514 & $U p^{a}$ \\
\hline 1814 & isovalerylglycine & 0.041 & 1. 517 & $U p^{a}$ \\
\hline 1945 & 5-hydroxymethyluracil & 0.022 & 1. 529 & Down $^{a}$ \\
\hline 2713 & tiopronin & 0.044 & 1.610 & Down $^{a}$ \\
\hline 2850 & kynurenic acid & 0.011 & 1.630 & $U p^{a}$ \\
\hline 2856 & 3-indolepropionic acid & 0.011 & 1.640 & $U p^{a}$ \\
\hline 2869 & glycyl-L-leucine & 0.039 & 1. 571 & $U p^{a}$ \\
\hline 2925 & $\mathrm{~N}$-acetyl-DL-methionine & 0.019 & 1.617 & $U p^{a}$ \\
\hline 3103 & 1, 3-dimethyluric acid & 0.025 & 1. 659 & $U p^{a}$ \\
\hline 4329 & $\mathrm{~N}$-acetyl-L-tyrosine & 0.018 & 1.641 & $U p^{a}$ \\
\hline 4374 & D-quinovose & 0.004 & 1.603 & $U p^{b}$ \\
\hline 5127 & L-gulonic gamma-lactone & 0.024 & 1. 521 & $U p^{a}$ \\
\hline 5361 & tetrahydrobiopterin & 0.037 & 1. 608 & Down $^{a}$ \\
\hline 5372 & pentadecanoic Acid & 0.005 & 1. 599 & Down ${ }^{b}$ \\
\hline 5531 & dioxybenzone & 0.045 & 1. 417 & $U p^{a}$ \\
\hline 5714 & quadrone & 0.000 & 1. 725 & Down ${ }^{b}$ \\
\hline 6094 & 5-hydroxymethylcytidine & 0.019 & 1. 619 & $U p^{a}$ \\
\hline 7596 & oleic acid & 0.032 & 1. 582 & Down $^{a}$ \\
\hline 7742 & stearic acid & 0.034 & 1. 542 & Down $^{a}$ \\
\hline 10198 & beta-Estradiol & 0.007 & 1.687 & Down ${ }^{b}$ \\
\hline 10469 & salidroside & 0.039 & 1. 582 & $U p^{a}$ \\
\hline 10588 & eicosapentaenoic Acid & 0.049 & 1. 501 & Down $^{a}$ \\
\hline 11683 & L-iditol & 0.044 & 1. 522 & Down $^{a}$ \\
\hline 13489 & ethynodiol diacetate & 0.016 & 1.637 & $U p^{a}$ \\
\hline 13779 & tamsulosin & 0.000 & 1. 707 & Down $^{b}$ \\
\hline 18005 & muramic acid & 0.000 & 1. 689 & Down $^{\text {b }}$ \\
\hline 24886 & deoxycholic acid & 0.006 & 1. 705 & $U p^{b}$ \\
\hline
\end{tabular}

Note: ${ }^{a}$ means significant difference, and ${ }^{\mathrm{b}}$ means extremely significant difference, Up means the fecal metabolites of captive North China leopard are higher than those in the wild, Down means the fecal metabolites of wild North China leopard are higher than those in the captive.

changes in normal microbes affect metabolic pathways. Due to the limited understanding of the gut microbiota of North China leopard, there are many unknown and future research will target diseased and healthy individuals in order to identify gut microbiota changes with potential relationships with diseases. This study provides constructive suggestions for the rational breeding of captive North China leopard and ideas for the rewilding and releasing of captive North China leopard from the perspective of the gut microbiota.

\section{Conclusion}

Our results suggested that gut microbiota compositions and fecal metabolic phenotypes of captive and wild North China leopard were significantly different, and found that some differentially abundant gut microbes were strongly correlated with changes in fecal metabolites.

To our knowledge, this study is the first to analyze and compare the gut microbiota and fecal metabolites of captive and wild North China leopard worldwide. The results of this study are helpful for zoos to develop better management strategies for captive leopard, and provide a theoretical basis for the breeding and reintroduction of North China leopard. With the continuous deepening of gut microbiata related research, it is expected to become an effective means to guide wild animal field monitoring and disease diagnosis.

\section{Management suggestions}

The single food of the captive North China leopard population affects the diversity of the gut microbiota; appropriate changes to the type of feed meat to be closer to the diet of the wild population would make the gut microbiota of the captive population closer to that of the wild population, which is conducive to the wild release of the captive population.

The low content of probiotics in the captive population is not conducive to better adaptation to the changeable climate. Therefore, some probiotics can be added during feeding to enrich the gut microbiota of the captive population.

Keeping the cage of the North China leopard population clean and hygienic will reduce the incidence of gut microbial infections, and once found problems can be dealt with in time.

The captive North China leopard bred by humans is not wild enough and lacks hunting skills. While the wild North China leopard lives alone, in order to survive in constant movement every day, the living area of the captive North China leopard can be set up to be larger, and living animals can be selected for feeding, thus preserving the predatory nature of the North China leopard and preparing for future wild development.

\section{Methods}

\section{Fecal sample collection}

Fecal sample collection of captive North China leopard

Three fresh fecal samples of captive North China leopard were randomly collected in Zhengzhou Zoo in Henan Province, China. Healthy leopard were randomly selected, and fecal samples were collected aseptically, 


\section{Statistics of Pathway Enrichment}

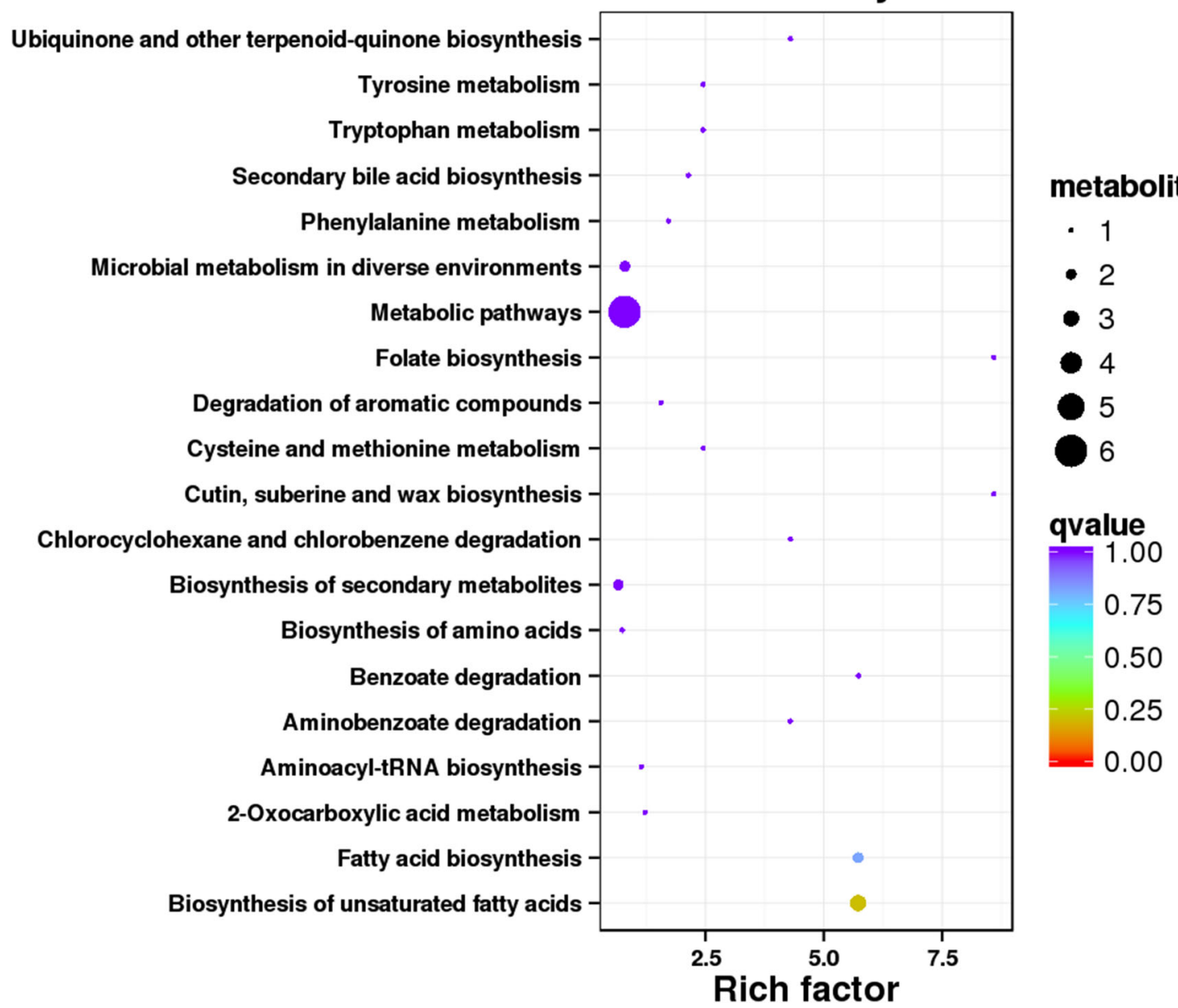

Fig. 5 Statistics of different metabolites on pathway enrichment. Note: The rich factor is the ratio of the proportion of different metabolites in the pathway and the proportion of all metabolites in the pathway. The larger the value is, the greater the enrichment degree is. Qvalue is Pvalue after multiple hypothesis test correction, and the closer it is to 0 , the more significant the enrichment is. The size of the points in the figure represents the number of metabolites enriched in the corresponding pathway

immediately after defecation. The sex, age, diets and health of captive leopard used in the experiment are shown in Table 2. The fresh fecal samples were sent to the laboratory on dry ice within 24 hours of collection and stored at $-80{ }^{\circ} \mathrm{C}$ for further microbial community analysis and metabolic analysis. We did not introduce any toxic substances that would interfere with the animal habitat. The research complied with the protocols established by the China Wildlife Conservation Association and the legal requirements of China.

\section{Fecal sample collection of wild North China leopard}

Three fresh wild leopard fecal samples were collected in the Tieqiaoshan Provincial Nature Reserve in Shanxi province, China, using disposable PE gloves and sealed bags. Wild leopard frozen feces were collected aseptically immediately after they were found during winter in the reserve. The collected feces samples were transported to the laboratory with the fastest possible speed on dry ice and stored at $-80^{\circ} \mathrm{C}$ for the next sequencing experiment. We use leopard species-specific primer and leopard distribution areas to determine whether the leopard was North China leopard. We did not introduce any toxic substances that would interfere with the animal habitat. The research complied with the protocols established by the China Wildlife Conservation Association and the legal requirements of China.

$16 \mathrm{~S}$ rRNA microbial community analysis

After extracting total DNA of the sample, a primer was designed according to the conserved region, a sequencing adapter was added at the end of the primer, polymerase chain reaction (PCR) amplification was carried out, and the product was purified, quantified and homogenized to form a sequencing library; the constructed library was first subjected to library quality inspection, 


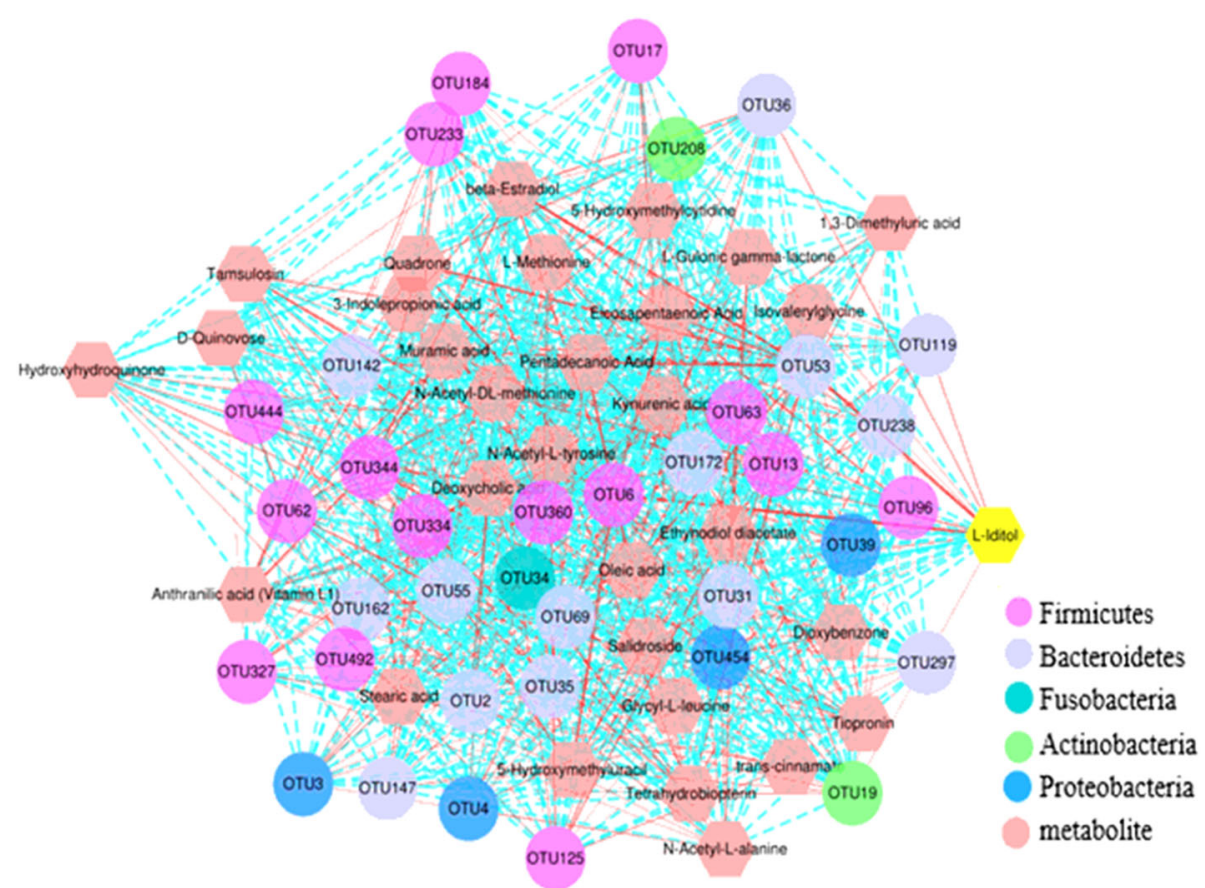

Fig. 6 The relationships between significantly different metabolites and differential abundant OTUs in the captive and wild North China leopard. A connection indicates that the OTUs have a correlation with the metabolite,a red solid line indicates a positive correlation, a blue dashed line indicates a negative correlation, and the line thickness indicates the strength of the correlation

and the library with qualified quality inspection was sequenced with the Illumina HiSeq 2500. The original data are spliced (FLASH, version 1.2.11), the spliced sequences were quality filtered (Trimmomatic, version 0.33), and chimeras (UCHIME, version 8.1) were removed to obtain high-quality tag sequences.

\section{Fecal metabolic profiling LC-MS analysis}

LC-MS/MS analyses were performed using an UHPLC system (1290, Agilent Technologies) with a UPLC BEH Amide column $(1.7 \mu \mathrm{m} 2.1 * 100 \mathrm{~mm}$, Waters) coupled to a TripleTOF 5600 (Q-TOF, AB Sciex). The mobile phase consisted of $25 \mathrm{~mm} \mathrm{NH4OAc}$ and $25 \mathrm{~mm} \mathrm{NH} 4 \mathrm{OH}$ in water $(\mathrm{pH}=9.75)(\mathrm{A})$, and acetonitrile (B) was carried with the elution gradient as follows:0 $\mathrm{min}, 95 \% \mathrm{~B} ; 7 \mathrm{~min}$, 65\% B;9 min, 40\% B;9.1 min, 95\% B;12 min, 95\% B, which was delivered at $0.5 \mathrm{ml} \mathrm{min}-1$. The injection volume was $3 \mu$ l. The Triple TOF mass spectrometer was used for its ability to acquire MS/MS spectra on an

Table 2 Sex, age, diet and health of captive leopards (A1, A4, A5) kept in Zhengzhou Zoo in Henan Province, China

\begin{tabular}{llcll}
\hline Sample & Sex & Age (year) & Condition & Diet \\
\hline A1 & Female & 1 & Healthy & Duck \\
A4 & Male & 4 & Healthy & Duck \\
A5 & Male & 5 & Healthy & Duck \\
\hline
\end{tabular}

information-dependent basis (IDA) during an LC/MS experiment. In this mode, the acquisition software (Analyst TF 1.7, AB Sciex) continuously evaluates the full scan survey MS data as it collects and triggers the acquisition of MS/MS spectra depending on preselected criteria. In each cycle, 12 precursor ions with intensity greater than 100 were chosen for fragmentation at a collision energy (CE) of $30 \mathrm{~V}$ ( $15 \mathrm{MS} / \mathrm{MS}$ events with a product ion accumulation time of $50 \mathrm{msec}$ each). ESI source conditions were set as follows: Ion source gas 1 at 60 Psi, Ion source gas 2 at 60 Psi, Curtain gas at 35 Psi, source temperature $650^{\circ} \mathrm{C}$, Ion Spray Voltage Floating(ISVF) $5000 \mathrm{~V}$ or $-4000 \mathrm{~V}$ in positive or negative mode, respectively [51].

\section{Statistical analysis}

Statistical comparisons were analyzed by the Statistical Package for Social Science program (SPSS22.0, Chicago, USA). $p<0.05$ was considered statistically significant.

\section{Abbreviations}

rRNA: Ribosomal ribonucleic acid; LC/MS: Liquid chromatography/mass spectrometer; EN: Endangered; E: Endangered; VU: Vulnerable; NT: Near Threatened; IUCN: International union for conservation of nature; CITE S: Convention on International Trade in Endangered Species of Wild Fauna and Flora; DNA: Deoxyribonucleic acid; PE: Polyethylene; OPLS -

DA: Orthogonal partial least squares - discrimination analysis; KEGG: Kyoto encyclopedia of genes and genomes; PCR: Polymerase Chain Reaction; UHPLC: Ultra High Performance Liquid Chromatography; NCBI: National center for biotechnology information; SRA: Short read archive 


\section{Acknowledgements}

We thank Pengfei Lang, Tao Xu and Wei Cao for sample collection. Thanks to Nathan for modifying the grammar of my article. Thanks to Xue Liang, Xin Liang, Zexu Long, Jingxuan Wang for their care and help.

\section{Authors' contributions \\ HC participated with the study design, bioinformatic analyses and manuscript preparation. JW carried out sample collection and sample processing. FH participated with statistical analyses and bioinformatic analyses. YH provided faecal materials and back ground information of leopards for the study and sample collection. GJ contributed analysis tools for the study and participated with bioinformatic analyses. YH and GJ collaborated in the design and coordination and helped to draft the manuscript. All authors read and approved the final manuscript.}

\section{Funding}

This research is funded by National Natural Science Foundation of China (NSFC 31872241), Fundamental Research Funds for the Central Universities (2572017PZ14), National Key Programme of Research and Development, Ministry of Science and Technology (2016YFC0503200), and Biodiversity Survey, Monitoring and Assessment Project of Ministry of Ecology and Environment, China (2019HB2096001006).The funders supported the charge of field studies, 16S rRNA sequencing, and metabolitics analysis.

\section{Availability of data and materials}

The datasets used and/or analysed during the current study are available from the corresponding author on reasonable request.

\section{Ethics approval and consent to participate}

Three fecal samples of the captive North China leopard were randomly collected with the permission of the authorities of Zhengzhou Zoo in Henan Province, China. And three wild leopard fecal samples were collected in the field, with the permission of the authorities of Tieqiaoshan Provincial Nature Reserve in Shanxi province, China. There is no conflict of interest with species conservation guidelines. All procedures related to animal's research were approved by the Ethics and Welfare of Experiment Animals Committee affiliated to Northeast Forestry University.

\section{Consent for publication}

Not applicable.

\section{Competing interests}

The authors declare that they have no competing interests.

\section{Author details}

${ }^{1}$ Feline Research Center of National Forestry and Grassland Administration, College of Wildlife and Natural Protected Area, Northeast Forestry University, 150040 Harbin, China. ${ }^{2}$ Guangdong Provincial Key Laboratory of Silviculture, Protection and Utilization, Guangdong Academy of Forestry, 510520 Guangzhou, China. ${ }^{3}$ College of Veterinary Medicine, Yunnan Agricultural University, 650201 Kunming, China.

\section{Received: 15 July 2020 Accepted: 18 September 2020} Published online: 29 September 2020

\section{References}

1. Dou H, Feng L, Xiao W, Wang T. The complete mitochondrial genome of the North China leopard (Panthera pardus japonensis). Mitochondrial Dna Part A Dna Mapping Sequencing Analysis. 2016;27:1167.

2. Laguardia A, Kamler JF, Li S, Zhang CC, Zhou ZF, Shi K. The current distribution and status of leopard Panthera pardus in China. Oryx. 2017;51: 153-9.

3. Han YL. Population Quantity Investigation of Panthera Pardus Fontanierii of Tieqiao Mountain in Shanxi. Shanxi Forestry Science Technology. 2018; 47(02):18-9.

4. Gong XY. Assessment on Habitat Suitability for Panthera Pardus Fontanierii in Tieqiao Mountain Nature Reserve Based on Maxent Model. Shanxi Forestry Science Technology. 2019;48(01):14-5 + 52.

5. Ding Y, Qi WU, Yibo HU, Wang X, Nie Y, Xiaoping WU, Wei F. Advances and prospects of gut microbiome in wild mammals. Acta Theriologica Sinica. 2017:37(4):399-406.
6. Sender R, Fuchs S, Milo R. Are We Really Vastly Outnumbered? Revisiting the Ratio of Bacterial to Host Cells in Humans. Cell. 2016;164:337-40.

7. Musso G, Gambino R, Cassader M. Gut microbiota as a regulator of energy homeostasis and ectopic fat deposition: mechanisms and implications for metabolic disorders. Curr Opin Lipidol. 2010;21:76-83.

8. Chen L, Zhang H, Liu G, Sha W. First report on the bacterial diversity in the distal gut of dholes (Cuon alpinus) by using 16S rRNA gene sequences analysis. J Appl Genet. 2016;57:275-83.

9. David LA, Maurice CF, Carmody RN, Gootenberg DB, Button JE, Wolfe BE, et al. Diet rapidly and reproducibly alters the human gut microbiome. Nature. 2014;505(7484):559.

10. Hale VL, Tan CL, Niu K, Yang Y, Zhang Q, Knight R, et al. Gut microbiota in wild and captive Guizhou snub-nosed monkeys, Rhinopithecus brelichi. American Journal of Primatology. 2019;81:e22989.

11. Guo W, Mishra S, Wang C, Zhang H, Ning R, Kong F, et al. Comparative Study of Gut Microbiota in Wild and Captive Giant Pandas (Ailuropoda melanoleuca). Genes. 2019;10:827.

12. Morris JG. Idiosyncratic nutrient requirements of cats appear to be dietinduced evolutionary adaptations. Nutr Res Rev. 2002;15:153-68.

13. Hooper LV, Midtvedt T, Gordon JI. How host-microbial interactions shape the nutrient environment of the mammalian intestine. Annu Rev Nutr. 2002 22:283-307.

14. De Filippo C, Cavalieri D, Di Paola M, Ramazzotti M, Poullet JB, Massart S, et al. Impact of diet in shaping gut microbiota revealed by a comparative study in children from Europe and rural Africa. Proc Natl Acad Sci USA. 2010;107:14691-6.

15. Nelson TM, Rogers TL, Carlini AR, Brown MV. Diet and phylogeny shape the gut microbiota of Antarctic seals: a comparison of wild and captive animals. Environ Microbiol. 2013;15:1132-45.

16. Steffens DC, Jiang W, Krishnan KR, Karoly ED, Mitchell MW, Connor CM,et al. Metabolomic differences in heart failure patients with and without major depression. journal of geriatric psychiatry neurology. 2010;23(2):138.

17. Raman M, Ahmed I, Gillevet PM, Probert CS, Ratcliffe NM, Smith S, et al. Fecal microbiome and volatile organic compound metabolome in obese humans with nonalcoholic fatty liver disease. Clinical gastroenterology hepatology: the official clinical practice journal of the American Gastroenterological Association. 2013;11(7):868.

18. Nicholson JK, Holmes E, Kinross J, Burcelin R, Gibson G, Jia W, et al. Host-gut microbiota metabolic interactions. Science. 2012;336:1262-7.

19. Liu M, Zhu J, Chen J, Ding H. GaoY, Characterization of Snow Leopard(Panthera uncia) Fecal Microbiota by Barcoded Pyrosequencing. Chinese Journal of Wildlife. 2019;40(04):873-81.

20. Zhang H, Liu G, Chen L, Sha W. Composition and diversity of the bacterial community in snow leopard (Uncia uncia) distal gut. Ann Microbiol. 2015; 65:703-11.

21. Chen L.Bacterial diversity in the intestinal tract of wolves and comparative and phylogenetic analysis of canidae mitochondrial genomes.Harbin: Northeast Forestry University,2010.

22. He F, Liu D, Zhang L, Zhai J, Ma Y, Xu Y, et al. Metagenomic analysis of captive Amur tiger faecal microbiome. BMC Veterinary Research. 2018;14: 379.

23. Zhang $\mathrm{H}$, Chen L. Phylogenetic analysis of $16 \mathrm{~S}$ rRNA gene sequences reveals distal gut bacterial diversity in wild wolves (Canis lupus). Mol Biol Rep. 2010;37:4013-22.

24. Han S, Guan Y, Dou H, Yang H, Yao M, Ge J, et al. Comparison of the fecal microbiota of two free-ranging Chinese subspecies of the leopard (Panthera pardus) using high-throughput sequencing. PeerJ. 2019;7:e6684.

25. Wasimuddin MS, Melzheimer J, Thalwitzer S, Heinrich S, Wachter B, et al. Gut microbiomes of free-ranging and captive Namibian cheetahs: Diversity, putative functions and occurrence of potential pathogens. Mol Ecol. 2017; 26:5515-27.

26. Chen L, Liu M, Zhu J, Gao Y, Chen JX, Sha WL. Diversity and sex-specific differences in the intestinal microbiota of cheetah (Acinonyx jubatus). Acta Microbiologica Sinica. 2019;59(09):1723-36.

27. Barry KA, Middelbos IS, Boler BMV, Dowd SE, Suchodolski JS, Henrissat B, et al. Effects of Dietary Fiber on the Feline Gastrointestinal Metagenome. J Proteome Res. 2012;11:5924-33.

28. Jia X. Study on diversity and function of interstinal bacterial flora from African lions of different seasons. Jilin Agricultural University,2017.

29. He F, Zhai J, Zhang L, Liu D, Ma Y, Rong K, et al. Variations in gut microbiota and fecal metabolic phenotype associated with Fenbendazole 
and Ivermectin Tablets by $16 \mathrm{~S}$ rRNA gene sequencing and LC/MS-based metabolomics in Amur tiger.Biochemical and Biophysical Research Communications. 2018;499:447-53.

30. Deng P, Swanson KS. Gut microbiota of humans, dogs and cats: current knowledge and future opportunities and challenges. Br J Nutr. 2015;113: 6-17.

31. An C, Okamoto Y, Xu S, Ko KY, Kimura J, Yamamoto N. Comparison of fecal microbiota of three captive carnivore species inhabiting Korea. J Vet Med Sci. 2017;79:542-6.

32. Lozupone CA, Stombaugh Jl, Gordon Jl, Jansson JK, Knight R. Diversity, stability and resilience of the human gut microbiota. Nature. 2012;489: 220-30.

33. Hildebrandt MA, Hoffmann C, Sherrillmix SA, Keilbaugh SA, Hamady M Chen $Y Y$, et al. High-fat diet determines the composition of the murine gut microbiome independently of obesity. Gastroenterology. 2009;137: $1716-24$.

34. Wu X, Zhang H, Chen J, Shang S, Yan J, Chen Y, et al. Analysis and comparison of the wolf microbiome under different environmental factors using three different data of Next Generation Sequencing. Scientific Reports. 2017;7.

35. Zhang DY, Hai-Feng Jl, Wei-Ling XU. The Effect of Probiotics on the Intestine Microbial Ecology of Animals. China Animal Husbandry Veterinary Medicine. 2007;34(3):15-8.

36. Hill DA, Artis D. Intestinal Bacteria and the Regulation of Immune Cell Homeostasis. Annu Rev Immunol. 2010;28:623-67.

37. Su Z, Li S, Zou G, Yu C, Sun Y, Zhang H, et al. Urinary metabonomics study of anti-depressive effect of Chaihu-Shu-Gan-San on an experimental model of depression induced by chronic variable stress in rats. J Pharm Biomed Anal. 2011;55:533-9.

38. Zheng P, Gao H, Li Q, Shao W, Zhang M, Cheng K, et al. Plasma Metabonomics as a Novel Diagnostic Approach for Major Depressive Disorder. J Proteome Res. 2012;11:1741-8.

39. Su Z, Jia H, Zhang $H$, Feng $Y$, An L, Zou Z. Hippocampus and serum metabolomic studies to explore the regulation of Chaihu-Shu-Gan-San on metabolic network disturbances of rats exposed to chronic variable stress. Mol Biosyst. 2014;10:549-61.

40. Le Gall G, Noor SO, Ridgway K, Scovell L, Jamieson C, Johnson IT, et al. Metabolomics of Fecal Extracts Detects Altered Metabolic Activity of Gut Microbiota in Ulcerative Colitis and Irritable Bowel Syndrome. J Proteome Res. 2011;10:4208-18.

41. Jia H, Feng Y, Liu Y, Chang $X$, Chen L, Zhang $H$, et al. Integration of $1 H$ NMR and UPLC-Q-TOF/MS for a Comprehensive Urinary Metabonomics Study on a Rat Model of Depression Induced by Chronic Unpredictable Mild Stress. Plos One. 2013;8:e63624.

42. Weir TL, Manter DK, Sheflin AM, et al. Stool Microbiome and Metabolome Differences between Colorectal Cancer Patients and Healthy Adults. Plos One. 2013;8(8):e70803.

43. Wu J, Yang JJ, Yang F, et al. Analysis of Alkaline and Neutral Volatile Metabolites in Feces by Gas Chromatography-Tandem Mass Spectrometry. Anal Chem. 2017:45(6):837-43.

44. Watanabe $\mathrm{H}$, Miyamoto $\mathrm{Y}$, Enoki $\mathrm{Y}$, et al. p-Cresyl sulfate, a uremic toxin, causes vascular endothelial and smooth muscle cell damages by inducing oxidative stress. Pharmacology Research \& Perspectives. 2015;3: e00092.

45. Hale VL, Chen J, Johnson S, et al. Shifts in the Fecal Microbiota Associated with Adenomatous Polyps. Cancer Epidemiology Biomarkers Prevention. 2016;26(1):85-94.

46. Magnuson AD, Guanchen L, Tao S, et al. Supplemental methionine and stocking density affect antioxidant status, fatty acid profiles, and growth performance of broiler chickens. J Animal Sci.2020;98:4.

47. Ghoochani BB, Aliannejad R, Oskouie AA, et al. Metabolomics diagnostic approach to mustard airway diseases: A preliminary study. Iranian Journal of Basic Medical Sciences. 2018;21(1):59-69.

48. Arrieta MC, Stiemsma LT, Dimitriu PA, Thorson L, Russell S, Yurist-Doutsch S, et al. Early infancy microbial and metabolic alterations affect risk of childhood asthma. Science Translational Medicine. 2015;307ra152.

49. Shi $X$, Wei $X$, Yin $X$, Wang $Y$, Zhang $M$, Zhao $C$, et al. Hepatic and Fecal Metabolomic Analysis of the Effects of Lactobacillus rhamnosus GG on Alcoholic Fatty Liver Disease in Mice. J Proteome Res. 2015;14: 1174-82.

50. Yen S, McDonald JAK, Schroeter K, Oliphant K, Sokolenko S, Blondeel EJM, et al. Metabolomic Analysis of Human Fecal Microbiota: A Comparison of
Feces-Derived Communities and Defined Mixed Communities. J Proteome Res. 2015;14:1472-82.

51. Wang HY, Zhao J, Zhang YQ. The flavonoid-rich ethanolic extract from the green cocoon shell of silkworm has excellent antioxidation, glucosidase inhibition, and cell protective effects in vitro. Food Nutrition Research. 2020;64: 1637.

\section{Publisher's Note}

Springer Nature remains neutral with regard to jurisdictional claims in published maps and institutional affiliations.
Ready to submit your research? Choose BMC and benefit from:

- fast, convenient online submission

- thorough peer review by experienced researchers in your field

- rapid publication on acceptance

- support for research data, including large and complex data types

- gold Open Access which fosters wider collaboration and increased citations

- maximum visibility for your research: over $100 \mathrm{M}$ website views per year

At BMC, research is always in progress.

Learn more biomedcentral.com/submissions 